\title{
De Haas-van Alphen effect in 2D systems: application to mono- and bilayer graphene
}

\author{
Igor A. Luk'yanchuk \\ University of Picardie Jules Verne, Laboratory of Condensed Matter Physics, Amiens, France; \\ L.D. Landau Institute for Theoretical Physics, Moscow, Russia \\ E-mail: lukyanc@ferroix.net \\ Received July 29, 2010
}

\begin{abstract}
Generalization of Shoenberg theory of de Haas-van Alphen magnetic oscillation in 2D metals for the case of electrons with arbitrary spectrum dispersion is used to propose the way to determine the nature of charge carriers in 2D conducting systems: mono- and bilayer graphene. We analyzed the analytical expression for the oscillating susceptibility as a function of chemical potential and distinguished its characteristic features for carriers with normal parabolic and Dirac-like linear spectra that can be detected experimentally.
\end{abstract}

PACS: 71.20.-b Electron density of states and band structure of crystalline solids;

71.18.+y Fermi surface: calculations and measurements; effective mass, $g$ factor;

81.05.U- Carbon/carbon-based materials.

Keywords: de Haas-van Alphen effect, graphene, 2D conducting systems.

Quantum oscillation of magnetic moment provided by electron Landau level (LL) quantization in strong magnetic field was firstly observed in Bismuth by de Haas and van Alphen (dHvA) in 1930 [1] almost simultaneously with Landau prediction [2]. Their first satisfactory theory description in simple metals was done during 30's by Peierls [3], Shoenberg [5] and Landau [6].

Further exploration of dHvA effect was related with understanding of temperature and impurities effects. Remarkable progress was achieved after the Lifshitz and Kosevich (LK) [7] generalization of Shoenberg and Landau theory for the arbitrary electron spectrum and Fermi surface (FS). Basing on Lifshitz-Onsager semiclassical quantization of the electron orbital motion in magnetic field, moving in $k$-space around FS with cross-section $S$

$$
S=(n+\gamma) 2 \pi \hbar \frac{e H}{c},
$$

LK demonstrated that only electrons having the extremal cross-section orbits give the contribution to dHvA oscillation and that $S$ is the most relevant parameter to describe the oscillation. In LK formalism the effective mass of electron is naturally generalized by the cyclotron mass, expressed through the derivative of $S$ over the chemical potential:

$$
m^{*}=\frac{1}{2 \pi} \frac{d S}{d \mu}
$$

DHvA effect measurements of $S$ as a function of field orientation and $\mu$ permitted to reconstruct the electronic dispersion spectrum in various metals and semi-metals $[8,9]$.

After incorporation of the impurity effect, described by the Dingle, LL broading $\Gamma=\hbar / 2 \tau[10]$ ( $\tau$ is the mean free time of electrons) the classical LK formula for the oscillated part of magnetization (denoted by tilde) looks like

$$
\begin{gathered}
\widetilde{M}_{3 D}=-\frac{\sqrt{2 \pi}}{8 \pi^{3}} \frac{\mu_{B}}{a_{B}^{3}}\left(\frac{S / m^{*}}{\mathrm{Ry}}\right)\left(\frac{\mu_{B} H}{\mathrm{Ry}}\right)^{1 / 2}\left|\frac{\partial^{2} S}{\partial p_{z}^{2}}\right|^{-1 / 2} \times \\
\times \sum_{l=1, \sigma= \pm 1}^{\infty} \frac{\psi(\lambda l)}{l^{3 / 2}} \mathrm{e}^{-\pi \beta \frac{\Gamma}{\mu_{B} H} l} \sin \left(\beta \frac{S / m^{*}}{2 \mu_{B} H} l-2 \pi \gamma_{\sigma} l \pm \frac{\pi}{4}\right),
\end{gathered}
$$

where the dimensionless term $\partial^{2} S / \partial p_{z}^{2}$ is the parallel to field curvature of FS at the extremal belts ( \pm is the sign of $\partial^{2} S / \partial p_{z}^{2}$ ), term $S / m^{*}$ has the dimensionality of the energy and

$$
\beta=\frac{m^{*}}{m}, \quad \lambda=\beta \frac{\pi^{2} T}{\mu_{B} H}, \quad \psi(x)=\frac{x}{\sinh x} .
$$

We assume here $k_{B}=1$ and use the atomic units: Bohr magneton, Bohr radius and Rydberg energy:

$$
\mu_{B}=\frac{e \hbar}{2 m c}, \quad a_{B}=\frac{\hbar^{2}}{m e^{2}}, \quad \mathrm{Ry}=\frac{m e^{4}}{2 \hbar^{2}} .
$$


Phase parameter $\gamma_{\sigma}$ includes both the Lifshitz-Onsager quantization parameter $\gamma$ from Eq. (1) and Zeeman spinsplitting factor $\sigma= \pm 1[11-12]$ :

$$
\gamma_{\sigma}=\gamma+\frac{1}{2} \beta \sigma
$$

It is interestingly that although the Peierls interpretation of dHvA effect [3] (1933) as intersection of Fermi level with LLs is more intuitively clear for 2D case with Dirac comb density of states, the initial theory was done for 3D metals where the calculations are more complicated because of account of $k_{z}$-dispersion of the spectra. The regular study of quantum oscillation in 2D systems was started only in 70's with the era of thin films, superlattices, MOSstructures and quantum wells.

In 1984 Shoenberg [13] proposed the formula for 2D dHvA oscillation in simplest case of parabolic spectrum with no impurity scattering in form of harmonic series. Further history of generalization of Shoenberg formula for 2D case repeated its 3D LK counterpart. In 2001 Champel and Mineev [14], basing on the Bychkov's formalism of Green's functions [15] calculated the dHvA oscillation for FS in form of corrugated cylinder that permits to follow the crossover from 3D LK metal to pure 2D system. The particular expression for 2D geometry was presented in elegant form that justifies the Shoenberg formula and includes the Dingle impurity scattering. Very recently we demonstrated [16] that Champel and Mineev formula can be written for the arbitrary electron spectra in the invariant LK terms of FS cross-section as

$$
\begin{gathered}
\tilde{M}_{2 D}=-\frac{1}{4 \pi^{3}} \frac{\mu_{B}}{a_{B}^{2}}\left(\frac{S / m^{*}}{\mathrm{Ry}}\right) \times \\
\times \sum_{l=1, \sigma= \pm 1}^{\infty} \frac{\psi(\lambda l)}{l} \mathrm{e}^{-\beta \frac{\pi \Gamma}{\mu_{B} H} l} \sin \left(\beta \frac{S / m^{*}}{2 \mu_{B} H}-2 \pi \gamma_{\sigma}\right) l .
\end{gathered}
$$

Although magnetic measurements are more complicated for 2D samples, the free from $k_{z}$ smearing dHvA signal in such systems can give much more information about the electronic process. Moreover, as it follows from comparison (3) and (7), dHvA oscillation in 2D geometry should be more intense because of the absence of the small factor $\left(\mu_{B} H / \mathrm{Ry}\right)^{1 / 2}$.

Intensive study of graphite monolayer - graphene started in 2005 after publications [17,18] challenges the exploration of quantum oscillation for understanding of $2 \mathrm{D}$ carbon nanostructures. Shubnikov-de Haas ( $\mathrm{SdH})$ oscillation of conductivity, oscillation of Nernst constant and quantum Hall effect were already discovered in graphene. Using the general formula (7) we shall discuss the possible application of dHvA effect in graphene. Although dHvA oscillation has not yet being detected in graphene, they can be more simple for interpretation then quantum oscillation of transport properties there.

The peculiarity of electrons in graphene is that, unlike normal carriers $(\mathrm{NC})$ with parabolic dispersion, classical dependence $S(\mu)$ and fixed cyclotron mass:

$$
\mathrm{NC}: \quad \varepsilon(p)=\frac{p^{2}}{2 m_{\perp}}, \quad S=2 \pi m_{\perp} \mu, \quad m^{*}=m_{\perp},
$$

they have the linear two-branch spectrum of so-called massless Dirac fermions (DF), quadratically dependent on $\mu$ FS cross-section and cyclotron mass that vanishes when chemical potential $\mu$ approaches to the Dirac point $\varepsilon=0$ :

$$
\text { DF: } \quad \varepsilon(p)= \pm v|p|, \quad S=\pi \frac{\mu^{2}}{v^{2}}, \quad m^{*}=\frac{\mu}{v^{2}} .
$$

More profound difference between NC and DF is coming in the phase factor $\gamma$ which is expressed via the Berry phase of the circulation of the matrix element

$$
\Omega(\mathbf{k})=i \int u_{\mathbf{k}}^{*}(r) \nabla u_{\mathbf{k}}(r) d^{2} r
$$

( $u_{\mathbf{k}}(r)$ is the periodic part of the electron Bloch function, the integration is done over a unit cell) around the electron orbit $\Gamma_{k}$ in $k$-space:

$$
\gamma=\frac{1}{2}-\frac{1}{2 \pi} \oint_{\Gamma_{k}} \Omega(\mathbf{k}) d \mathbf{k} .
$$

Factor $\gamma$ is the topologically protected quantum number [19] and

$$
\gamma=0 \text { for } \mathrm{DF}, \text { and } \gamma=\frac{1}{2} \text { for } \mathrm{NC} \text {. }
$$

Phase measurement of $\gamma$, firstly realized in 2004 [16] to distinguish the charge carriers in graphite, was successfully used in $2005[17,18]$ as the principal method to detect the DF in graphene.

We discuss here how the unconventional DF dependencies of LK parameters $S$ and $m^{*}$ on $\mu$ in graphene can be manifested in dHvA effect. The natural laboratory for the dHvA $\mu$-dependence exploration would be the substratedeposited graphene in which the gate voltage drives the variation of the chemical potential. We would like to stress that such setup pins the chemical potential by the gate voltage and the effect of dHvA oscillation of $\mu$ itself [8] does not appear here. To compare the results for DF in graphene with those for $\mathrm{NC}$ one can use the bilayer graphene [20] that according to band calculation has the two-branch parabolic spectrum $\varepsilon(p)= \pm p^{2} / 2 m_{\perp}$ and NC-type of LK parameters.

Equation (7) can be simplified at low temperatures $\pi^{2} \beta T<\mu_{B} H$ when $\lambda<1$ and $\psi(\lambda l) \approx 1$. For typical for graphene $m^{*}<0.02 m$ and $H=10 \mathrm{~T}$ this takes $T<10 \mathrm{~K}$. 
Because of small $\mathrm{m}^{*}$ we neglect also the Zeeman splitting in $\gamma_{\sigma}$. Then, series (7) can be calculated exactly giving

$$
\begin{gathered}
\widetilde{M}_{2 D}=-\frac{1}{2 \pi^{3}} \frac{\mu_{B}}{a_{B}^{2}}\left(\frac{S / m^{*}}{\mathrm{Ry}}\right) \times \\
\times \sin \left(\beta \frac{S / m^{*}}{2 \mu_{B} H}-2 \pi \gamma\right) \\
\exp \left(\beta \frac{\pi \Gamma}{\mu_{B} H}\right)-\cos \left(\beta \frac{S / m^{*}}{2 \mu_{B} H}-2 \pi \gamma\right)
\end{gathered}
$$

In case of NC Eq. (13) was obtained by Champel and Mineev [14] and in case of DF by Sharapov, Gusynin and Beck [21].

We can also present the magnetic susceptibility $\tilde{\chi}_{2 D}=\left(\partial \widetilde{M}_{2 D} / \partial H\right)_{\mu, T}$ as

$$
\begin{gathered}
\tilde{\chi}_{2 D}=\frac{\alpha^{2} a_{B}}{32 \pi^{3}} \beta\left(\frac{S / m^{*}}{\mu_{B} H}\right)^{2} \times \\
\times\left[\frac{\sinh \left(\beta \frac{\pi \Gamma}{\mu_{B} H}\right)}{\cosh \left(\beta \frac{\pi \Gamma}{\mu_{B} H}\right)-\cos \left(\beta \frac{S / m^{*}}{2 \mu_{B} H}-2 \pi \gamma\right)}-1\right],
\end{gathered}
$$

where $\alpha=e^{2} / \hbar c=1 / 137$ is the fine structure constant.

Equations (13), (14) are valid for any spectrum and permit to compare the dHvA dependencies $\widetilde{M}_{2 D}(\mu)$ and $\tilde{\chi}_{2 D}(\mu)$ both for DF in graphene and for $\mathrm{NC}$ in bilayer graphene as shown in Fig. 1.

In both cases the oscillation $\widetilde{M}_{2 D}(\mu)$ has the same characteristic inverted sawtooth shape, predicted by Shoenberg for the system with chemical potential $\mu$ driven by the external conditions [13] but several other features can distinguish two dependencies. The oscillation maxima in $\tilde{\chi}_{2 D}(\mu)$ and corresponding odd zeroes in

$\widetilde{M}_{2 D}(\mu)$ are equally distributed for NC:

$$
\mu_{n}=2 \mu_{B} H \frac{m}{m_{\perp}}\left(n+\frac{1}{2}\right),
$$

whereas they become more condensed at high $\mu$ for DF:

$$
\mu_{n}=\left[4 m v^{2} \mu_{B} H n\right]^{1 / 2}
$$

Another important feature is the different shape of the envelope function that increases in case of NC and has the nonmonotonous behavior in case of DF. To study this question in detail we consider the different limit cases.

In clean limit $\pi \beta \Gamma<\mu_{B} H$ the oscillating part of susceptibility can be present as the series of Lorentzians

$$
\tilde{\chi}_{2 D}=\chi_{\max }(\mu) \sum_{n} \frac{\Gamma^{2}}{\Gamma^{2}+\left(\mu-\mu_{n}\right)^{2}}+\chi_{\min }(\mu)
$$

confined between

$$
\chi_{\max }(\mu)=\frac{\alpha^{2} a_{B}}{16 \pi^{3}}\left(\frac{S / m^{*}}{\mu_{B} H}\right)^{2}\left(\frac{\mu_{B} H}{\pi \Gamma}\right)
$$

and

$$
\chi_{\min }(\mu)=-\frac{\alpha^{2} a_{B}}{64 \pi^{3}}\left(\frac{S / m^{*}}{\mu_{B} H}\right)^{2} .
$$

Specifying these dependencies for DF (9) and NC (8) we obtain that in both cases $\chi_{\max }, \chi_{\min }$ depend on the chemical potential in the similar way $\sim \mu^{2}$ and therefore are indistinguishable.

The situation changes in dirty limit $\pi \beta \Gamma>\mu_{B} H$ when

$$
\tilde{\chi}_{2 D}=\frac{\alpha^{2} a_{B}}{16 \pi^{3}} \beta\left(\frac{S / m^{*}}{\mu_{B} H}\right)^{2} e^{-\beta \frac{\pi \Gamma}{\mu_{B} H}} \cos \left(\beta \frac{S / m^{*}}{2 \mu_{B} H}-2 \pi \gamma\right)
$$
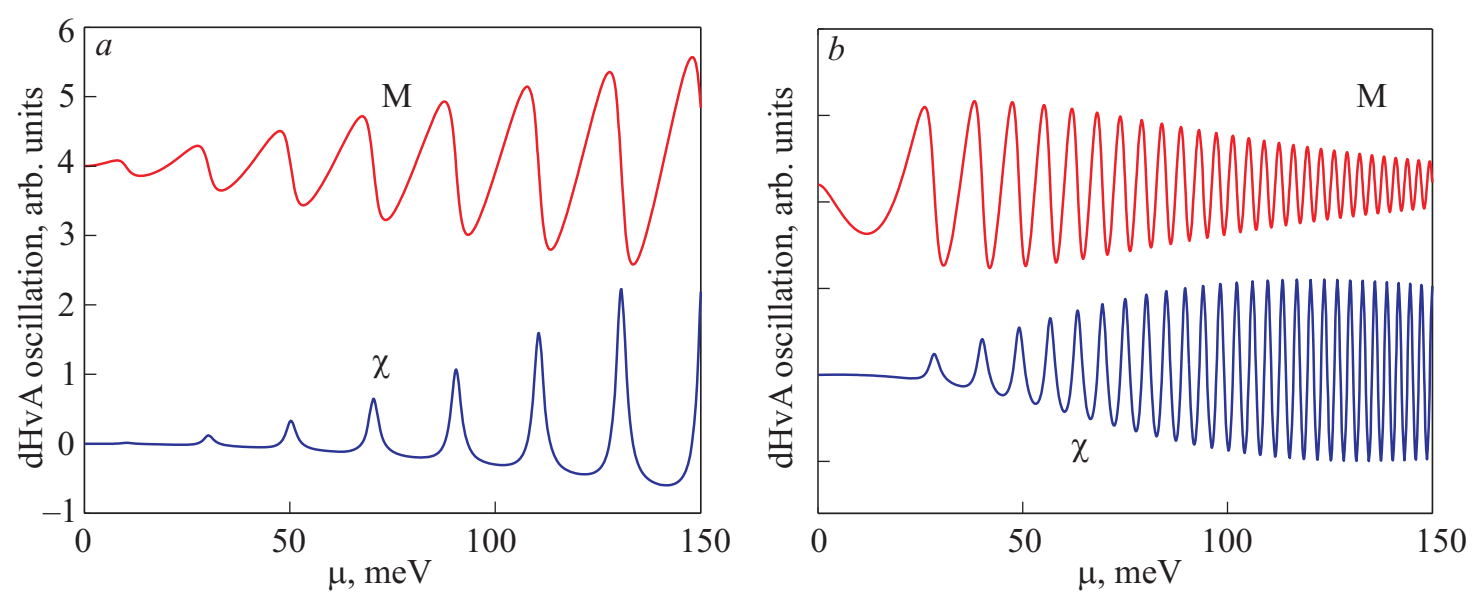

Fig. 1. 2D dHvA oscillation of magnetization $(M)$ and susceptibility $(\chi)$ at $H=3 \mathrm{~T}$ for normal carriers $(a)$ and for Dirac fermions $(b)$ as the function of chemical potential $\mu$. The effective mass of NC $m_{\perp}=0.02 \mathrm{~m}$, the velocity of DF $v=10^{8} \mathrm{~cm} / \mathrm{s}$. In both cases the Dingle temperature $\Gamma=15 \mathrm{~K}$. 
and the $\mu$-dependence of oscillating susceptibility is driven by the exponential factor $\exp \left[-\beta\left(\pi \Gamma / \mu_{B} H\right)\right]$. For $\mathrm{NC}$ with $\beta=m / m_{\perp}$ the exponential factor does not depend on $\mu$ and $\tilde{\chi}_{2 D}(\mu)$ always increases as $\mu^{2}$ because of the pre-exponent factor. For DF with $\beta=\mu / m v^{2}$ the exponential factor is decreasing function of $\mu$ and $\tilde{\chi}_{2 D}(\mu)$ first increases as $\mu^{3}$ because of the pre-exponential growth and then decays exponentially. This experimentally detectable difference between NC and DF explains the shape of the dHvA oscillations for NC and DF in Fig. 1.

The behavior of dHvA oscillation at high temperatures $\pi^{2} \beta T>\mu_{B} H$ when $\lambda<1$ and $\psi(\lambda l) \approx 2 \lambda l \exp (-\lambda l)$ is somewhat similar to the low- $T$ dirty limit since only the first term in the series expansion (7) can be kept and the formula for oscillating magnetization and susceptibility can be presented as

$$
\begin{gathered}
\widetilde{M}_{2 D}=-\frac{1}{\pi} \frac{\mu_{B}}{a_{B}^{2}} \beta\left(\frac{S / m^{*}}{\mathrm{Ry}}\right)\left(\frac{T}{\mu_{B} H}\right) \times \\
\times \exp \left(-\pi \beta \frac{\Gamma+\pi T}{\mu_{B} H}\right) \sin \left(\beta \frac{S / m^{*}}{2 \mu_{B} H}-2 \pi \gamma\right), \\
\tilde{\chi}_{2 D}=\frac{\alpha^{2}}{4 \pi} a_{B}\left(\frac{\beta T}{\mu_{B} H}\right) \beta\left(\frac{S / m^{*}}{\mu_{B} H}\right)^{2} \times \\
\times \exp \left(-\pi \beta \frac{\Gamma+\pi T}{\mu_{B} H}\right) \cos \left(\beta \frac{S / m^{*}}{2 \mu_{B} H}-2 \pi \gamma\right) .
\end{gathered}
$$

The amplitude of the oscillation however is substantially reduced by the exponential factor.

To conclude we generalized the Shoenberg theory of 2D dHvA oscillation for the case of the electrons with arbitrary spectrum and studied the oscillation of magnetic susceptibility as function of chemical potential for the electrons having the parabolic spectrum of normal carriers and linear spectrum of Dirac fermions, making the special emphasis on the features that permit to distinguish between these cases.

Note that the proposed theoretical description of $\mathrm{dHvA}$ oscillation is more simple and rigorous than that for $\mathrm{SdH}$ conductivity oscillation since it does not include the scattering kinetic effects. At the same time the experimental situation is exactly the opposite. The $\mathrm{SdH}$ oscillation were the first experimental method to study the DF in graphene systems $[17,18]$ whereas dHvA effect is not yet observed there. Possibly the best compromise between theoretical and experimental tools would be the observed giant quantum oscillation of the Nernst coefficient $[22,23]$, the theory of which was recently developed in [24] and that actually stimulated the current work. At the same time the $\mu$-dependence of the dHvA effect can be studied in the gated nano-thick layers of graphite where the existence of the DF was proposed $[16,25,26]$.
This work was made in frame of FP7-IRSES programs ROBOCON and TERACAN and was also supported by the French ANR Grant BLAN07-03-192276. Author thanks to A. Varlamov and A. Kavokin for the numerous discussions and suggestions.

1. W.J. de Haas and P.M. van Alphen, Proc. Netherlands Roy. Acad. Sci. 33, 680 (1930).

2. L.D. Landau, Z. Phys. 64, 629 (1930).

3. R. Peierls, Z. Phys. 80, 763 (1933).

4. R. Peierls, Z. Phys. 81, 186 (1933).

5. D. Shoenberg, Proc. Roy. Soc. A170, 341 (1939).

6. L.D. Landau, Proc. Roy. Soc. A170, 341 (1939) (Appendix to article of D. Shoenberg [5]).

7. I.M. Lifshitz and A.M. Kosevich, Zh. Éksp. Teor. Fiz. 29, 730 (1955).

8. D. Shoenberg, Magnetic Oscillations in Metals, Cambridge University Press (1984).

9. I.M. Lifshitz, M.Ya. Azbel', and M.I. Kaganov, Electron Theory of Metals, Consultants Bureau, New York (1973).

10. R.B. Dingle, Proc. Roy. Soc. A211, 500 (1952); ibid. A211, 517 (1952).

11. A. Akhieser, C. R. Acad. Sci. URSS 23, 874 (1939).

12. E.H. Sondheimer and A.H. Wilson, Proc. Roy. Soc. A210, 173 (1954).

13. D. Shoenberg, J. Low Temp. Phys. 56, 417 (1984)

14. T. Champel and V.P. Mineev, Philos. Mag. B81, 55 (2001).

15. Yu.A. Bychkov, Soviet. Phys. JETP 12, 977 (1961).

16. I.A. Luk'yanchuk and Y. Kopelevich, Phys. Rev. Lett. 93, 166402 (2004).

17. K.S. Novoselov, A.K. Geim, S.V. Morozov, D. Jiang, M.I. Katsnelson, I.V. Grigorieva, S.V. Dubonos, and A.A. Firsov, Nature 438, 197 (2005).

18. Y. Zhang, Y.-W. Tan, H.L. Stormer, and Philip Kim, Nature 438, 201 (2005).

19. G.P. Mikitik and Yu.V. Sharlai, Phys. Rev. Lett. 82, 2147 (1999).

20. K.S. Novoselov, E. McCann, S.V. Morozov, V.I. Fal'ko, M.I. Katsnelson, U. Zeitler, D. Jiang, F. Schedin, and A.K. Geim, Nature Physics 2, 177 (2006).

21. S.G. Sharapov, V.P. Gusynin, and H. Beck, Phys. Rev. B69, 075104 (2004).

22. Y.M. Zuev, W. Chang, and P. Kim, Phys. Rev. Lett. 102, 096807 (2009).

23. J.G. Checkelsky and N.P. Ong, Phys. Rev. B80, 081413(R) (2009).

24. A. Varlamov, A. Kavokin, and I. Lukyanchuk, to be published (2010).

25. I.A. Luk'yanchuk and Y. Kopelevich, Phys. Rev. Lett. 97, 256801 (2006).

26. I. Luk'yanchuk, Y. Kopelevich, and M. El Marssi, Physica B404, 404 (2009). 